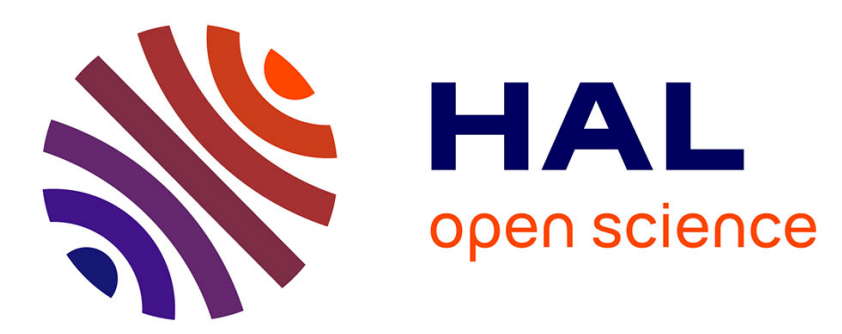

\title{
Development of Hand Preference for Object-Directed Actions and Pointing Gestures: A Longitudinal Study between 15 and 25 months of Age
}

Hélène Cochet

\section{- To cite this version:}

Hélène Cochet. Development of Hand Preference for Object-Directed Actions and Pointing Gestures: A Longitudinal Study between 15 and 25 months of Age. Developmental Psychobiology, 2011, 54 (1), pp.105-111. 10.1002/dev.20576 . halshs-01464324

\section{HAL Id: halshs-01464324 \\ https://shs.hal.science/halshs-01464324}

Submitted on 11 Jul 2017

HAL is a multi-disciplinary open access archive for the deposit and dissemination of scientific research documents, whether they are published or not. The documents may come from teaching and research institutions in France or abroad, or from public or private research centers.
L'archive ouverte pluridisciplinaire HAL, est destinée au dépôt et à la diffusion de documents scientifiques de niveau recherche, publiés ou non, émanant des établissements d'enseignement et de recherche français ou étrangers, des laboratoires publics ou privés. 


\section{Brief Report}

\section{Hélène Cochet \\ Department of Psychology \\ Research Center in the Psychology \\ of Cognition, Language and Emotion \\ Aix-Marseille University \\ 29, av. Robert Schuman \\ 13621 Aix-en-Provence Cedex 1, France \\ E-mail: helene.cochet@univ-provence.fr \\ Development of Hand Preference for Object-Directed Actions and Pointing Gestures: A Longitudinal Study between 15 and 25 months of Age}

\begin{abstract}
The development of hand preferences for object-directed actions and pointing gestures was investigated in toddlers sampled bimonthly between 15 and 25 months of age. Language level was also assessed, in an attempt to examine the relationship between handedness and language development. Results did not reveal any changes over the study period in the mean Handedness Index of the whole sample, both for bimanual manipulative activities and pointing gestures. However, the categorization of participants as left-handers, right-handers, or non-lateralized revealed that most of children presented nonlinear individual trajectories in the development of hand preference. Moreover, the only significant correlations observed between hand preferences for manipulation and pointing were negative correlations between the strength of hand preferences at 19 and 21 months of age, suggesting that manipulative actions and communicative gestures are controlled by different networks in the left cerebral hemisphere. These findings are discussed in relation to the development of speech-gesture links in infancy. (c) 2011 Wiley Periodicals, Inc. Dev Psychobiol 54: 105-111, 2012.
\end{abstract}

Keywords: handedness; bimanual manipulation; pointing gestures; language development

\section{INTRODUCTION}

Both speech and gestures are associated with left-hemispheric asymmetries in adults (e.g., Knecht et al., 2000), but the relationship between the emergence of language lateralization and manual asymmetries in infants is still unclear. In an attempt to answer this question, the present study aimed at exploring the relationship between hand preference in different activities

Received 3 January 2011; Accepted 5 May 2011

Correspondence to: $\mathrm{H}$. Cochet

Contract grant sponsor: French National Research Agency (ANR)

Contract grant number: ANR-08-BLAN-0011 01

Published online 8 June 2011 in Wiley Online Library (wileyonlinelibrary.com). DOI 10.1002/dev.20576

(C) 2011 Wiley Periodicals, Inc. and language development in toddlers, over a 10-month period during the second year of life.

It is now widely acknowledged that infants begin to use gestures to communicate before they use words, and the role of gestures in speech development has been highlighted in several studies (see Colonnesi, Stams, Koster, \& Noom, 2010). In addition, researchers have shown not only that infants' pointing gestures were predominantly produced with the right hand (e.g., Blake, O'Rourke, \& Borzellino, 1994; Cochet \& Vauclair, 2010a; Young, Lock, \& Service, 1985), but also that this right-sided asymmetry was stronger than the one reported for noncommunicative manual actions (Bates, O'Connell, Vaid, Sledge, \& Oakes, 1986; Cochet \& Vauclair, 2010b; Vauclair \& Imbault, 2009). Moreover, laterality for both unimanual and bimanual manipulative actions appears not to be significantly correlated (e.g., Cochet \& Vauclair, 2010c; Esseily, 
Jacquet, \& Fagard, 2011), or to be only weakly correlated with laterality for communicative gestures (Vauclair \& Imbault, 2009).

These findings suggest, first, that manual asymmetries for gestures and for manipulative activities follow distinct developmental trajectories in the course of ontogeny. Second, considering both the early signs of left hemisphere specialization for language processing in infants (e.g., Dubois et al., 2009; Friederici, Friedrich, \& Christophe, 2007; Mills, Coffey-Corina, \& Neville, 1993) and the role of gestures in language learning (e.g., Camaioni, Perucchini, Bellagamba, \& Colonnesi, 2004; Goldin-Meadow, 2006), it can be hypothesized that the emergence of manual asymmetries for gestures is linked to the left-hemispheric specialization for language. However, few studies have investigated the development of hand preferences in relation to language acquisition by distinguishing communicative gestures from manipulative actions. Recent research revealed that the frequency of right-handed pointing gestures in 14-month-old infants was correlated to the number of words understood and to the number of words produced, while no significant relation was found between handedness for grasping and language level (Esseily et al., 2011). The relationship between hand preference and language now needs to be investigated throughout development, as this relationship is likely to change at critical periods of speech acquisition (e.g., Bates et al., 1986).

Longitudinal studies also prove to be necessary to compare the strength and stability of hand preferences for noncommunicative activities and communicative gestures across development. Signs of right-sided asymmetries are already expressed in infancy, but the consistency in the degree of hand preference for manipulative activities appears quite limited during the infant's first year of life, especially in unimanual prehension tasks (e.g., Corbetta \& Thelen, 1999; McCormick \& Maurer, 1988; Ramsay, 1985). Bimanual activities, in which each hand has a specific and different role from the other hand, are now regarded as more reliable measures of handedness, as they require more lateralized patterns of actions (Fagard \& Lockman, 2005; Fagard \& Marks, 2000). The distinction between active and passive roles for the two hands has been widely exemplified with the tube task, in which the non-dominant hand grasps a tube while the dominant hand picks up the object or food inserted in it (e.g., Hopkins et al., 2005; Vauclair \& Imbault, 2009). The proportion of right-handed children in bimanual tasks has been reported to increase between 10 and 40 months of age (Vauclair \& Imbault, 2009), however, other researchers did not observe any changes in the degree of handedness in both unimanual and bimanual activities between 13 and 28 months (Bates et al., 1986) and between 18 and 36 months of age (Fagard \& Marks, 2000). With regard to communicative gestures, findings from several studies focusing on pointing gestures have suggested that the right-sided asymmetry is established in early stages of development. Indeed, the degree of right-hand preference was found to increase between prepointing produced at 8 months of age and later pointing produced at 15 months (Young et al., 1985) and 12 months of age (Blake et al., 1994). By contrast, studies with older children did not report any increase in the right-sided bias for pointing between approximately 1 and 3 years of age (Bates et al., 1986; Cochet \& Vauclair, 2010a; Vauclair \& Imbault, 2009).

Overall, these results indicate that the development of hand preference for both bimanual manipulative activities and communicative gestures deserves further investigation. The aim of the present study was to examine the dynamic relationship between language acquisition and hand preferences for bimanual manipulative activities and pointing gestures. Children were followed up every 2 months between 15 and 25 months of age, in their home. First, we expected to observe different patterns of hand preference between object-directed actions and pointing gestures, in favor of a stronger asymmetry for pointing. Second, we expected the development of hand preference for pointing to be more closely related to language, compared to handedness for manipulation.

\section{METHOD}

\section{Participants}

Eight French children (four girls and four boys), recruited in daycare centers, were studied every 2 months in their home with their mother between 15 and 25 months of age. Due to house moving or other family events, two additional children only participated in the first session and were therefore not included in the study. The data were missing for another child at 23 months of age due to illness. Insofar as it concerned only one of the six sessions, this child was not excluded from the study. All children were from middle- to upper-middleclass monolingual French-speaking families.

\section{Procedure}

Each session began with a short warming-up period, in the child's bedroom or in the living-room. The language test, two bimanual manipulation tasks and two pointing tasks were then administered in an alternated order across participants. For both manipulative activities and pointing gestures, an attempt was made to administer the same number of trials in each of the two tasks. However, more trials could be administered in one task if children were reluctant to perform the 
other one. Children were seated either at a child-size table or on the floor, depending on what they were more accustomed to. The experimenter was seated in front of the participants and showed them different objects and pictures. The mother stayed in the room throughout each session, but she was instructed not to initiate interactions with the child and not to point toward the different targets during testing. For the assessment of hand preference, data were only collected when children were sitting in a symmetrical posture, with both hands initially free before starting a trial. All sessions were videotaped.

Language Assessment. The "language" subtest of the French Brunet-Lézine scale (1965) revised by Josse (1997) was used to measure language level. This scale assesses psychomotor development between 2 and 30 months of age. The language subtest comprises a task in which children have to identify familiar objects $(n=10$, including for example a spoon and a pair of glasses) and pictures ( $n=15$, including for example a banana and a bike). Participants either directly name the different items or point toward the objects and pictures designated by the experimenter when they are too young to produce the corresponding words (in the latter case, children had to point to a specific picture among either 6 or 9 different pictures, depending on their age). Other items are based on parental reports ( $n=9$, for the age range studied), but most of the time, the experimenter could also observe the target behavior during the session (e.g., knowing whether children use their first name when talking about themselves). Both language production and comprehension are thus assessed, although the total raw score obtained does not allow distinguishing between these different language components. The maximum possible raw score is 73. A developmental age for language is inferred from the raw score via the available French norms. Dividing the developmental age by the chronological age yields a developmental quotient (DQ) for language.

Manipulative Tasks. For both tasks, the hand playing an active role was considered as the dominant hand and the one having a role of support or orientation as the non-dominant hand. In the bottle task, children had to hold a small transparent plastic bottle $(6 \mathrm{~cm}$ in diameter) with their non-dominant hand and take out the stuffed toy that was placed in it with their dominant hand. Five different stuffed toys were used. In the column task, children had to remove a plastic ring from a Fisher-Price column with their dominant hand. The experimenter made sure that the ring was pushed down just far enough to require children to hold the base of the column with their non-dominant hand. In total, children undertook between 5 and 10 trials, depending on their willingness to accomplish the tasks.

Pointing Tasks. In the first task, children were asked to point to different pictures (e.g., a ball, an elephant) in a children's book positioned in front of them, either on the table or on the floor. In the second task, children had to point to toys that had been positioned in front of them by the experimenter, at a distance of approximately $1.5 \mathrm{~m}$ away. Between 5 and 15 trials were administered in total, depending on children's willingness to produce pointing gestures. Our initial objective was to perform a number of trials sufficient to record at least 8 pointing gestures for each child, without exceeding 15 trials, however, so as to keep the children's attention and interest. Nevertheless, as pointing gestures proved to be relatively difficult to elicit in some children over the study period, we reduced the minimum threshold to 5 pointing gestures. This number of trials, although limited, is sufficient to reliably assess hand-preference patterns, as indicated by other studies conducted in toddlers (e.g., Cochet \& Vauclair, 2010b; Vauclair \& Imbault, 2009). Moreover, Handedness Index (HI) scores (see below) for manipulative actions and pointing gestures could still be calculated with a comparable number of responses. The mean numbers of manipulative activities and pointing gestures recorded for each child are displayed in Table 1.

\section{Data Analyses}

An individual HI score was calculated for each participant using the formula $(R-L) /(R+L)$, where $R$ and $L$ stand for the total right- and left-hand responses. The HI values lay along a continuum from -1 to 1 , with the sign indicating hand-preference direction and the absolute value reflecting hand-preference strength. A $Z$-score was also calculated using the formula $(R-L) /$ square $(R+L)$ (Fagard \& Lemoine, 2006; Michel, Sheu, \& Brumley, 2002), in order to classify participants as right-handers $(Z \geq 1.65)$, left-handers $(Z \leq 1.65)$, or non-lateralized $(-1.65<Z<1.65)$.

\section{Reliability}

The experimenter coded all the sessions from the video recordings. A second coder who was blind to the hypotheses of the study coded $21 \%$ of the total number of sessions (10 sessions were randomly selected). Significant and strong correlations were found between the reliability coding and the main coding for language score, $r=.97, p<.01$, handedness score for manipulation $r=.99, p<.01$, and hand preference score for pointing, $r=.83, p<.01$, thus indicating high level of inter-rater reliability.

Table 1. Mean Numbers of Bimanual Manipulative Activities and Pointing Gestures (and Standard Deviations) Recorded for Each Child

\begin{tabular}{|c|c|c|c|c|c|c|c|c|}
\hline Participants & Nat. & Cla. & Val. & Ele. & Giu. & Ani. & Jon. & Ada. \\
\hline Bima & $17(41)$ & $7.5(1.22)$ & $8.17(.41)$ & & $8.2(.4$ & & $7.83(.41)$ & $7.33(2.07)$ \\
\hline Pointing gestures & $8.33(.82)$ & $8.0(.0)$ & $8.67(1.03)$ & $8.33(.82)$ & $8.4(.68)$ & $8.67(.47)$ & $9.0(1.10)$ & $8.0(1.41)$ \\
\hline
\end{tabular}




\section{RESULTS}

\section{Language Development}

The mean language scores and the mean DQs were calculated for each session (see Tab. 2). Friedman ANOVA revealed a significant increase in language raw scores between 15 and 25 months of age, $\chi^{2}(n=7$; $d f=5)=34.9 ; \quad p<.001 \quad$ (Wilcoxon matched-pairs signed rank tests indicated that all two-by-two adjacent age differences were significant). There was no significant changes in the mean DQ for language between 15 and 25 months of age, $\chi^{2}(n=7 ; d f=5)=4.31$; $p=.51$.

\section{Development of Hand Preference}

First, we examined the development of hand preference through the analysis of Mean Handedness Indexes (MHI) of the whole sample (see Tab. 2). Friedman ANOVA did not reveal any changes in hand preference between 15 and 25 months of age, either for manipulative activities, $\chi^{2}(n=7 ; d f=5)=6.96 ; p=.22$, or for pointing gestures, $\chi^{2}(n=7 ; d f=5)=4.10$; $p=.53$.

Therefore, in order to provide a global evaluation of infants' hand preference, we calculated single handedness scores for each participant grouping all sessions together. MHI for bimanual manipulation varied between -.41 and $1.0(M=.49 ; S D=.46)$ and MHI for pointing gestures varied between -.19 and .86 $(M=.53 ; S D=.35)$. In the manipulation task, six children were classified as right-handers, one as lefthander and one as non-lateralized. In the pointing task, seven children were classified as right-handers and one child as non-lateralized.

Second, we investigated the stability of hand preference for manipulative activities and pointing gestures focusing on the categorization of children as left-handers, right-handers, or non-lateralized over the 10-month period of the study (see Tab. 3). Only one child was strictly right-handed for manipulative activities over the six sessions. With regard to pointing gestures, two children were strictly right-handed over the six sessions. When the classification did not remain stable over the study period, several scenarios were observed, but no clear developmental shifting patterns emerged. Children shifted either once or several times in hand preference, either being first classified as right-handers or non-lateralized. However, it can be noted that no child was classified as left-hander before 19 months of age in the manipulation task, and before 21 months of age in the pointing task.

\section{Relation between Hand Preference for Pointing and Manipulation}

Considering the global hand preference scores (i.e., all sessions grouped together), the categorization as righthanders was consistent across the manipulation and pointing tasks for six of the eight children. One child was classified as non-lateralized in both tasks and the last child was classified as right-hander in the pointing task and as left-hander in the manipulation task. There was no significant difference in the MHI for pointing gestures and manipulative activities, $Z=.28 ; p=.78$, $n=8$, and no significant correlation between these different measures of hand preference, $r=.31 ; p=.46$.

Further analyses considering each session separately also failed to reveal any significant differences between MHI for pointing gestures and manipulative activities. Moreover, none of the correlations between the two measures of hand preference was significant, whatever age was considered. However, correlational analyses performed on the absolute values of HI revealed significant negative correlations between the strength of hand preferences for manipulative actions and pointing gestures at 19 months $(r=-.79 ; p=.019)$ and at 21 months $(r=-.87 ; p=.005)$.

Furthermore, considering the six sessions separately, the categorization of children as left-handers, righthanders, or non-lateralized was consistent across the two tasks in 21 of the 47 overall observations, including 13 observations where children were right-handed both in the pointing task and the manipulative task, six observations where they were non-lateralized and two observations where they were left-handed.

Table 2. Mean Language Scores, Mean Developmental Quotients, and Mean Handedness Indexes (MHI) for Bimanual Manipulative Activities and Pointing Gestures at Each Age (in Months)

\begin{tabular}{lcrrrrr}
\hline & 15 months & 17 months & 19 months & 21 months & 23 months & 25 months \\
\hline Raw score & $24.4(3.8)$ & $31.1(4.5)$ & $38.8(6.8)$ & $45.8(10.6)$ & $58.1(9.8)$ & $63.8(10.6)$ \\
Developmental quotient & $95.1(14.0)$ & $102.0(8.2)$ & $103.4(9.5)$ & $102.7(14.0)$ & $110.0(12.6)$ & $107.6(14.3)$ \\
MHI bimanual manipulation & $.78(.36)$ & $.54(.47)$ & $.59(.70)$ & $.21(.80)$ & $.28(.65)$ & $.52(.37)$ \\
MHI pointing gestures & $.63(.23)$ & $.53(.60)$ & $.50(.42)$ & $.62(.55)$ & $.33(.68)$ & $.51(.47)$ \\
\hline
\end{tabular}

Figures in brackets refer to standard deviations. 
Table 3. Distribution of Participants as Right-Handers, Left-Handers, or Non-Lateralized for Manipulative Activities and Pointing Gestures as a Function of Age (in Months)

\begin{tabular}{|c|c|c|c|c|c|c|}
\hline & 15 months & 17 months & 19 months & 21 months & 23 months & 25 months \\
\hline \multicolumn{7}{|c|}{ Manipulative activities } \\
\hline Right-handers & 6 & 3 & 6 & 3 & 2 & 4 \\
\hline Left-handers & 0 & 0 & 1 & 2 & 2 & 0 \\
\hline Non-lateralized & 2 & 5 & 1 & 3 & 3 & 4 \\
\hline \multicolumn{7}{|l|}{ Pointing gestures } \\
\hline Right-handers & 4 & 5 & 4 & 6 & 3 & 5 \\
\hline Left-handers & 0 & 0 & 0 & 1 & 1 & 1 \\
\hline Non-lateralized & 4 & 3 & 4 & 1 & 3 & 2 \\
\hline
\end{tabular}

\section{Relation between Hand Preference and Language Development}

Spearman's correlations were performed to investigate the relation between language level and handedness over development. At 15, 17, 19, 23, and 25 months of age, neither raw language scores nor DQs were correlated to $\mathrm{HI}$ for pointing gestures or for manipulative activities. At 21 months of age, results revealed a negative correlation between raw language score and HI for pointing gestures, $r=-.73 ; p=.042$, whereas language score was not correlated with HI for manipulative activities, $r=.46 ; p=.25$.

Finally, Mann-Whitney $U$ tests were used to assess the potential influence of gender on the different variables studied (HI scores and language level), after applying Bonferroni corrections to adjust the level of significance. There was no difference between boys and girls on any of the variables, whatever age was considered.

\section{DISCUSSION}

The present study confirmed earlier evidence for the existence of right-sided asymmetries in young children (e.g., Bates et al., 1986; Blake et al., 1994; Cochet \& Vauclair, 2010a,b; Young et al., 1985), thus demonstrating the preferential involvement of the left cerebral hemisphere both in the control of noncommunicative actions and pointing gestures. Results did not reveal any changes between 15 and 25 months of age in the MHI of the whole sample for bimanual manipulative activities or pointing gestures, in line with several studies with children of comparable age ranges (Bates et al., 1986; Cochet \& Vauclair, 2010a; Fagard \& Marks, 2000; Vauclair \& Imbault, 2009). However, we cannot exclude the possibility that bimonthly sampling intervals did not allow us to observe transient and subtle variations of hand preference. For example, Ferre, Babik, and Michel (2010) have shown that developmental changes in the degree of hand preference for object prehension could only be identified using monthly sampling intervals.

Moreover, the calculation of mean indexes tends to mask interindividual variability, whereas the latter needs to be considered in order to identify potentially distinct trajectories in the development of hand preferences. Thus, at the individual level, some children exhibited stable hand-use preferences for bimanual activities and pointing gestures over the ten months of the study, while others presented nonlinear trajectories in handedness development. The classification of participants as left-handers, right-handers, or non-lateralized revealed that most of children shifted several times in preference over the study period, but no clear developmental shifting patterns emerged from our results. However, children mainly shifted from right-hander to non-lateralized, or from non-lateralized to right-hander, reflecting fluctuations in the strength rather than in the direction of hand preferences throughout development, both for object manipulation and pointing.

The comparison of hand preferences for noncommunicative actions and pointing gestures did not reveal any significant difference in MHI between the two types of activities, contrary to results of previous studies reporting a stronger right-sided bias for pointing (Bates et al., 1986; Esseily et al., 2011; Vauclair \& Imbault, 2009). However, as previously mentioned, MHI does not take into account individual variations during development. Further analyses showed that the two measures of hand preference were not significantly correlated, whatever age was considered, which supports previous findings of studies that examined the relationship between hand preferences for pointing and unimanual reaching (Cochet \& Vauclair, 2010b; Esseily et al., 2011). Moreover, strong negative correlations were observed between the strength of hand preferences (i.e., the absolute values of $\mathrm{HI}$ ) for manipulative actions and pointing gestures at 19 and 21 months of age, indicating that strongly lateralized children in the pointing task were weakly lateralized in the 
manipulative task, and conversely. The distinction between left-handed, right-handed, and non-lateralized children at each session also showed that the classification was different across both tasks in more than $55 \%$ of observations.

These different developmental trajectories are consistent with the hypothesis according to which a bimodal communication system, specialized for both gestural and vocal communication, is distinct from the system controlling noncommunicative motor functions in the left cerebral hemisphere (e.g., Gentilucci, \& Dalla Volta, 2008; Özyürek, Willems, Kita, \& Hagoort, 2007; $\mathrm{Xu}$, Gannon, Emmorey, Smith, \& Braun, 2009). The fact that the strength of hand preferences varied in opposite directions at 19 months and at 21 months of age might be related to specific periods in speech development likely to influence left hemispheric activity, in particular the lexical spurt period. The lexical spurt is a strong increase in lexical production occurring between 18 and 22 months of age, once children's vocabulary size reaches about 50 words (e.g., Goldfield \& Reznick, 1990; Nazzi \& Bertoncini, 2003). A recent study reported that the lexical spurt was accompanied by an increase in the degree of hand preference for pointing gestures, but not for bimanual manipulative activities (Cochet, Jover, \& Vauclair, 2011). Therefore, this sudden change in the rate of word learning may be associated with high mobilization of the bimodal communication system in the left cerebral hemisphere, while fewer resources may be attributed to the purely motor system during the same period. In line with this hypothesis, the number of children categorized as righthanders in the pointing task was found to increase between 19 and 21 months of age, while it decreased in the bimanual manipulation task. Although the language test used in the present study did not allow us to determine precisely the onset of the lexical spurt for each participant, these results support the association between increasing lateralization for pointing gestures and increasing productive vocabulary (Cochet et al., 2011; Esseily et al., 2011).

However, several limitations of the present study need to be mentioned. First, the language scale used did not distinguish between language comprehension and production, whereas hand preference might relate in different ways to these different functions. Second, the low number of participants did not allow us to identify common patterns of changes in manual laterality across children, in relation to language development, and restricts the generalization of our findings regarding the relationship between hand preferences for manipulation and pointing at 19 and 21 months of age. Future studies with larger samples and additional measures of language level may shed light on some unexpected results such as the negative correlation between hand preference for pointing and language level at 21 months of age, and should enable to investigate further whether the emergence of manual asymmetries for gestures is linked to the left-hemispheric specialization for speech.

\section{NOTES}

The author would like to thank Jacqueline Fagard for her indepth reading and comments on the manuscript. This research was supported by a French National Research Agency (ANR) grant, reference ANR-08-BLAN-0011_01.

\section{REFERENCES}

Bates, E., O'Connell, B., Vaid, J., Sledge, P., \& Oakes, L. (1986). Language and hand preference in early development. Developmental Neuropsychology, 2, 1-15.

Blake, J., O'Rourke, P., \& Borzellino, G. (1994). Form and function in the development of pointing and reaching gestures. Infant Behavior and Development, 17, 195203.

Brunet, O., \& Lézine, I. (1965). Le développement psychologique de la première enfance [Psychological development in infancy]. Paris: Presses Universitaires de France.

Camaioni, L., Perucchini, P., Bellagamba, F., \& Colonnesi, C. (2004). The role of declarative pointing in developing a theory of mind. Infancy, 5, 291-308.

Cochet, H., Jover, M., \& Vauclair, J. (2011). Hand preference for pointing gestures and bimanual manipulation around the vocabulary spurt period. Journal of Experimental Child Psychology (in press). DOI: 10.1016/j.jecp.2011. 04.009

Cochet, H., \& Vauclair, J. (2010a). Features of spontaneous pointing gestures in toddlers. Gesture, 10(1), 86-107.

Cochet, H., \& Vauclair, J. (2010b). . Pointing gestures produced by toddlers from 15 to 30 months: Different functions, handshapes and laterality patterns. Infant Behavior and Development, 33, 432-442.

Cochet, H., \& Vauclair, J. (2010c). Pointing gesture in young children: Hand preference and language development. Gesture, 10(2/3), 129-149.

Colonnesi, C., Stams, G., Koster, I., \& Noom, M. J. (2010). The relation between pointing and language development: A meta-analysis. Developmental Review, 30(4), 352-366.

Corbetta, D., \& Thelen, E. (1999). Lateral biases and fluctuations in infants' spontaneous arm movements and reaching. Developmental Psychobiology, 34(4), 237-255.

Dubois, J., Hertz-Pannier, L., Cachia, A., Mangin, J. F., Le Bihan, D., \& Dehaene-Lambertz, G. (2009). Structural asymmetries in the infant language and sensori-motor networks. Cerebral Cortex, 19(2), 414-423.

Esseily, R., Jacquet, A. Y., \& Fagard, J. (2011). Handedness for grasping objects and pointing and the development of 
language in 14-month-old infants. Laterality (in press). DOI: 10.1080/1357650X.2010.499911.

Fagard, J., \& Lemoine, C. (2006). The role of imitation in the stabilization of handedness during infancy. Journal of Integrative Neuroscience, 5(4), 519-533.

Fagard, J., \& Lockman, J. (2005). The effect of task constraints on infants' (bi)manual strategy for grasping and exploring objects. Infant Behavior and Development, 28, 305-315.

Fagard, J., \& Marks, A. (2000). Unimanual and bimanual tasks and the assessment of handedness in toddlers. Developmental Science, 3(2), 137-147.

Ferre, C. L., Babik, I., \& Michel, G. F. (2010). Development of infant prehension handedness: A longitudinal analysis during the 6- to 14-month age period. Infant Behavior and Development, 33(4), 492-502.

Friederici, A. D., Friedrich, M., \& Christophe, A. (2007). Brain responses in 4-month-old infants are already language specific. Current Biology, 17, 1208-1211.

Gentilucci, M., \& Dalla Volta, R. (2008). Spoken language and arm gestures are controlled by the same motor control system. The Quarterly Journal of Experimental Psychology, 61, 944-957.

Goldfield, B. A., \& Reznick, S. J. (1990). Early lexical acquisition: Rate, content, and the vocabulary spurt. Journal of Child Language, 17, 171-183.

Goldin-Meadow, S. (2006). Talking and thinking with our hands. Current Directions in Psychological Science, 15, 34-39.

Hopkins, W. D., Russell, J., Freeman, H., Buehler, N., Reynolds, E., \& Schapiro, S. J. (2005). The distribution and development of handedness for manual gestures in captive chimpanzees (Pan troglodytes). Psychological Science, 16, 487-493.

Josse, D. (1997). Brunet-Lézine révisé: Echelle de développement psychomoteur de la première enfance [Revised Brunet-Lézine: Scale of psychomotor development in infancy]. Issy-les-Moulineaux: EAP.
Knecht, S., Dräger, B., Deppe, M., Bobe, L., Lohmann, H., Flöel, A., Ringelstein, B. E., \& Henningsen, H. (2000). Handedness and hemispheric language dominance in healthy humans. Brain, 123, 2512-2518.

McCormick, C. M., \& Maurer, D. M. (1988). Unimanual hand preferences in six-month-olds: Consistency and relation to familial-handedness. Infant Behavior and Development, 11, 21-29.

Michel, G. F., Sheu, C. F., \& Brumley, M. R. (2002). Evidence of a right-shift factor affecting infant hand-use preferences from 7 to 11 months of age as revealed by latent class analysis. Developmental Psychobiology, 40(1), 1-13.

Mills, D. L., Coffey-Corina, S. A., \& Neville, H. J. (1993). Language acquisition and cerebral specialization in 20month-old infants. Journal of Cognitive Neuroscience, 5, 317-334.

Nazzi, T., \& Bertoncini, J. (2003). Before and after the vocabulary spurt: Two modes of word acquisition? Developmental Science, 6(2), 136-142.

Özyürek, A., Willems, R. M., Kita, S., \& Hagoort, P. (2007). On-line integration of semantic information from speech and gesture: Insights from event-related brain potentials. Journal of Cognitive Neuroscience, 19, 605-616.

Ramsay, D. S. (1985). Fluctuations of unimanual hand preference in infants following the onset of duplicated syllable babbling. Developmental Psychology, 21, 318-324.

Vauclair, J., \& Imbault, J. (2009). Relationships between manual preferences for manipulative actions and for pointing gestures in infants and toddlers. Developmental Science, 12, 1060-1069.

Xu, J., Gannon, P. J., Emmorey, K., Smith, J. F., \& Braun, A. R. (2009). Symbolic gestures and spoken language are processed by a common neural system. Proceedings of the National Academy of Sciences of the United States of America, 106(49), 20664-20669.

Young, A. M., Lock, A. J., \& Service, V. (1985). Infants' hand preferences for actions and gestures. Developmental Neuropsychology, 1, 17-27. 